\title{
Polymer mesh scaffold combined with cell-derived ECM for osteogenesis of human mesenchymal stem cells
}

\author{
Yong Kwan Noh ${ }^{1,2}$, Ping Du', In Gul Kim', Jaehoon $\mathrm{Ko}^{3}$, Seong Who Kim ${ }^{4}$ and Kwideok Park ${ }^{1,5^{*}}$
}

\begin{abstract}
Background: Tissue-engineered scaffold should mimic the structure and biological function of the extracellular matrix and have mechanically supportive properties for tissue regeneration. In this study, we utilized a PLGA/PLA mesh scaffold, coated with cell-derived extracellular matrix (CDM) and assessed its potential as an osteogenic microenvironment for human umbilical cord blood-derived mesenchymal stem cells (UCB-MSCs). CDM was obtained by decellularization of in vitro-cultured type I collagen overexpressing (Col I -293 T-DK) cells. Test groups are mesh itself (control), fibronectin-coated (FN-mesh), and CDM-coated mesh scaffold (CDM-mesh). CDM was then solubilized and used for scaffold coating.

Results: CDM was successfully collected and applied to mesh scaffolds. The presence of CDM was confirmed via SEM and FN immunofluorescence. After then, UCB-MSCs were seeded into the scaffolds and subjected to the induction of osteogenic differentiation for 21 days in vitro. We found that the seeded cells were viable and have better proliferation activity on CDM-mesh scaffold. In addition, when osteogenic differentiation of UCB-MSCs was examined for up to 21 days, alkaline phosphatase (ALP) activity and osteogenic marker (COL I, ALP, osteocalcin, bone sialoprotein) expression were significantly improved with UCB-MSCs when cultured in the CDM-mesh scaffold compared to the control and FN-mesh.
\end{abstract}

Conclusion: Polymer mesh scaffold incorporated with CDM can provide UCB-MSCs with a better microenvironment for osteogenesis in vitro.

Keywords: Osteogenesis, Cell-derived extracellular matrix (CDM), Polymer mesh scaffold, Umbilical cord bloodderived mesenchymal stem cells (UCB-MSCs), Microenvironment

\section{Background}

Bone tissue engineering has an ultimate goal to regenerate damaged or lost bone tissue via osteoconductive and/or osteoinductive scaffolds [1]. The 3D polymer scaffolds is supposed to provide appropriate microenvironments to support stem cells adhesion, growth and differentiation, making them suitable for a new bone formation [2]. To do this, polymer scaffolds have been combined with biomaterials derived from natural sources. Examples are collagen [3], gelatin [4], fibrin [5], silk fibroin, keratin [6], and

\footnotetext{
* Correspondence: Kpark@kist.re.kr

${ }^{1}$ Center for Biomaterials, Korea Institute of Science and Technology, Seoul 02792, Republic of Korea

${ }^{5}$ Department of Biomedical engineering, University of Science and

Technology, Daejeon 34113, Republic of Korea

Full list of author information is available at the end of the article
}

others [7]. In addition, extracellular matrix (ECM) is a complex network of a variety of proteins, proteoglycans, and other macromolecules, where it can provide structural and biochemical support to the surrounding cells $[8,9]$. It is well recognized that ECM microenvironments are critical to support cell adhesion, migration, proliferation, and differentiation [10]. Therefore, many studies have also utilized ECM as a valuable resource in tissue engineering [11]. Specifically, ECM obtained from in vitro cultured cells has been studied as a source of bone tissue engineering $[12,13]$. Cell-derived extracellular matrix (CDM) promotes osteogenic differentiation of preosteoblasts and bone marrow mesenchymal stromal cells, respectively [14]. In addition CDMs are obtained from various cell types and their positive effects are investigated on the 
multi-lineage differentiation of human mesenchymal stromal cells [15].

However few studies have examined the effect of cellderived ECM in combination with an engineered 3D scaffold. From this perspective, we have developed a new platform, composed of a biodegradable PLGA/PLA mesh scaffold, functionalized with bioactive CDM derived from type I collagen overexpressing (Col I -293 T-DK) cells. Our hypothesis is that CDM coated polymer mesh scaffold can represent 3D microenvironment suitable for MSCs adhesion, proliferation, and osteogenic differentiation. In this work, we selected umbilical cord bloodderived mesenchymal stem cells (UCB-MSCs) as a MSCs source. Like other MSCs, UCB-MSCs possess a high proliferation rate for in vitro expansion and have multi-potency capable of differentiating into osteogenic, chondrogenic, and adipogenic lineage $[16,17]$. In our study, we found out a significant improvement of osteogenesis of UCB-MSCs on CDM-treated mesh scaffold.

\section{Methods}

Preparation of PLGA/PLA mesh scaffolds

Poly (L-lactide-co-glycolide) (PLGA; lactic to glycolic acid molar ratio, 50:50) and poly (L-lactide) (PLA) was purchased from EVONIK. PLGA and PLA fibers, 2$2.5 \mathrm{~mm}$ in length, were prepared by using a rotary cutter and their nonwovens were produced via modified wet-laid process. PLGA and PLA fibers were mixed in an aqueous solution with a dispersing agent (1 wt.\% Pluronic F127; Sigma-Aldrich) and randomly laid on a wire mesh to filter the liquid. The formed web was subsequently processed through a thermal bonding, in which the web was transferred to a heater and cured at $170{ }^{\circ} \mathrm{C}$ for $5 \mathrm{~min}$. The resulting mesh was cut into sheets $(4 \times 4 \times 4$ $\mathrm{mm}, \mathrm{L} \times \mathrm{W} \times \mathrm{H})$ and they were sterilized by soaking in $100 \%$ ethanol under ultraviolet (UV) light.

\section{Cell-derived matrix (CDM) preparation}

Collagen type I-overexpression cell line (Col I-293 TDK) was cultured at the density of $1.3 \times 10^{4}$ cells $/ \mathrm{cm}^{2}$ in a $100 \mathrm{~mm}$ diameter petri-dish for 4 days in the Dulbecco's Modified Eagle's Medium (DMEM) supplemented with $10 \%$ fetal bovine serum (FBS) and 100 $\mathrm{U} / \mathrm{mL}$ penicillin and $100 \mu \mathrm{g} / \mathrm{mL}$ streptomycin $(\mathrm{P} / \mathrm{S})$. At the time of confluence, the cell culture plates were washed twice with phosphate buffered saline (PBS), incubated briefly in a detergent solution containing $0.25 \%$ Triton $\mathrm{X}-100$ and $10 \mathrm{mM} \mathrm{NH}_{4} \mathrm{OH}$ (Sigma-Aldrich) at $37^{\circ} \mathrm{C}$, and then subjected to the treatment of $50 \mathrm{U} / \mathrm{mL}$ DNase I and $2.5 \mu \mathrm{L} / \mathrm{mL}$ RNase A (Invitrogen) for $1 \mathrm{~h}$ at $37{ }^{\circ} \mathrm{C}$. After the decellularization process, the specimens were washed with PBS thoroughly and stored at $4{ }^{\circ} \mathrm{C}$ before use.

\section{CDM characterization: protein and DNA content}

For CDM analysis, DNA was examined from the organic phase using Trizol $^{\circledR}$ reagent (Invitrogen). $0.3 \mathrm{~mL}$ of $100 \%$ $\mathrm{EtOH}$ was added to isolate the DNA from each sample and after centrifugation the supernatant was collected. The supernatant was washed twice with $0.1 \mathrm{M}$ sodium citrate in $10 \% \mathrm{EtOH}$, then centrifuged at $2000 \mathrm{~g}$ for $5 \mathrm{~min}$ at $4{ }^{\circ} \mathrm{C}$. The samples were resuspended in $2 \mathrm{~mL}$ of $75 \%$ ethanol and centrifuged again. The pellets were dissolved in $300 \mu \mathrm{L}$ of $8 \mathrm{mM} \mathrm{NaOH}$ and subsequently quantified using a NanoDrop ND 1000 Spectrophotometer (Thermo Fisher Scientific). In addition, BCA protein assay kit (23250, Thermo Scientific) was used according to the manufacturer's instructions to assess the total protein amount of CDM.

\section{Preparation of CDM-coated mesh scaffolds}

After the decellularization, CDM was harvested by gentle pipetting, transferred to $50 \mathrm{~mL}$ tubes, and vigorously agitated using a homogenizer (HG-3000, SMT, Japan) until a homogeneous aqueous phase was formed. The polymer mesh scaffolds were then immersed into the CDM suspension solution with a mild agitation and incubated for $24 \mathrm{~h}$. The CDM-coated mesh scaffolds were then freezedried overnight. Fibronectin (FN; BD Biosciences)-coated mesh scaffolds were also prepared by soaking the scaffolds in FN solution $\left(50 \mu \mathrm{g} / \mathrm{mL}\right.$ in distilled water) at $37{ }^{\circ} \mathrm{C}$ for $1 \mathrm{~h}$. The FN-coated scaffolds were then rinsed with distilled water and freeze-dried. The surface morphology of the FN- and CDM-coated mesh scaffolds was observed via scanning electron microscopy (SEM; Phenom G2 Pro Desktop). In addition, the distribution of the CDM in the mesh scaffolds was visualized via immunofluorescence staining of fibronectin using mouse monoclonal antibody (SC-8422; Santa Cruz Biotechnology) and Alex Fluor 488conjugated secondary antibody (goat anti-mouse IgG; Invitrogen), respectively.

\section{In vitro culture of UCB-MSCs and proliferation assay}

Human umbilical cord blood mesenchymal stem cells (UCB-MSCs) were kindly provided by MEDIPOST Co (Seoul, Korea). UCB-MSCs were cultured in Minimum Essential Medium alpha medium ( $\alpha$-MEM) supplemented with $10 \mathrm{FBS}$ and $1 \% \mathrm{P} / \mathrm{S}$. Cells were seeded at $5 \times 10^{5}$ cells in culture flasks and maintained at $37{ }^{\circ} \mathrm{C}$ in a humidified $5 \% \mathrm{CO}_{2}$ atmosphere with a medium change twice a week. Passage 9 UCB-MSCs were used throughout the experiments. Mesh scaffolds were transferred into non-adherent 24-well tissue culture plates, onto which UCB-MSCs were slowly inoculated at a density of $5 \times 10^{4}$ cells per scaffold. Cells were allowed to adhere for $3 \mathrm{~h}$ and cultured in a growth medium for up to 5 days. Three different test groups ( $n=3$, per group) were prepared: 1$)$ plain mesh (control), 2) fibronectin-coated mesh (FN-mesh), and 3) CDM-coated mesh (CDM-mesh). Cell proliferation was 
evaluated on $2^{\text {nd }}$ and $5^{\text {th }}$ day of culture using CCK-8 assay (Dojindo, Japan). Aliquots from each sample $(100 \mu \mathrm{L})$ were transferred into a 96-well plate and measured for the absorbance at a wavelength of $450 \mathrm{~nm}$ using a Multiskan microplate reader (Thermo Scientific).

\section{Osteogenic differentiation of UCB-MSCs}

Osteogenic differentiation of UCB-MSCs was induced in the presence of osteogenic supplements such as $10 \% \mathrm{FBS}, 1 \% \mathrm{P} / \mathrm{S}, 50 \mu \mathrm{g} / \mathrm{mL}$ ascorbic acid, $0.01 \mathrm{M}$ glycerol-2-phosphate, $50 \mathrm{ng} / \mathrm{mL}$ bone morphogenetic protein (BMP)-2 and $100 \mathrm{nM}$ dexamethasone for 1 and 3 weeks, respectively. Medium was changed every 2 or 3 day.

\section{Alkaline phosphatase (ALP) activity}

ALP activity of each group ( $n=4$ per group) after osteogenic induction for 3 weeks was analyzed using a Lab Assay ALP kit (Wako Pure Chemicals, Japan). Samples were incubated in the lysis buffer $(0.1 \%$ Triton X-100 in PBS) for $30 \mathrm{~min}$ at $37^{\circ} \mathrm{C}$. $50 \mathrm{~mL}$ of the lysis solution was added to $2 \mathrm{mg} / \mathrm{mL}$ of p-nitrophenyl phosphate (Sigma 104 tablet) in $0.1 \mathrm{M}$ Tris- $\mathrm{HCl}$ buffer ( $\mathrm{pH}$ 8.5). The absorbance was measured at $405 \mathrm{~nm}$ and normalized to the total amount of proteins in each sample lysate, which was assessed via BCA assay (Thermo Scientific).

\section{Quantitative real-time polymerase chain reaction (q-PCR)} Gene expression of osteogenic markers, such as bone sialoprotein (BSP), collagen type I (Col I), ALP, and osteocalcin (OC) was analyzed via quantitative realtime PCR. Total RNA was isolated using a Trizol $^{\circ}$ reagent (Invitrogen) extraction method. The extracted samples were subsequently quantified using a NanoDrop ND1000 Spectrophotometer (Thermo Fisher Scientific). cDNA synthesis was performed using a Maxime RT premix kit (Intron). All polymerase chain reactions were carried out using ABI Prism 7500 (Applied Biosystems) and gene expression level was quantified using SYBR Green (RR420A, TaKaRa). Relative gene expression level was calculated by the delta delta Ct method. The primer sequences of the target genes are as follows BSP: CAACCACCCTCTTCACCACT (forward) and GA TCTTCTGGGGTGGTCTCA (reverse); ALP: ATGGGA TGGGTGTT CCTACA (forward) and GTCTTAGAGA GGGCGACGTG (reverse); Col I: CAAGAACCC CAAG GACAAGA (forward) and GAATCCATCGGTCATG CTCT (reverse); OC: CCAGTT CTGCTCCTCTCCAG (forward) and GCCCACAGATTCCTCTTCTG (reverse) Housekeeping gene is glyceraldehyde-3-phosphate dehydrogenase (GAPDH): GGGCTCTCCAGAACATCATC (forward) and TTCTAGACGGCAGGTCAGGT (reverse).

\section{Histological analysis}

Harvested samples at 1 and 3 weeks were fixed in $4 \%$ paraformaldehyde for $24 \mathrm{~h}$, dehydrated, embedded in paraffin wax, and cut into $10 \mu \mathrm{m}$ thickness. Those thin sections ( $n=4$ per group) were then subjected to Alizarin Red, ALP and von kossa staining, respectively.

\section{Statistical analysis}

Data are expressed as mean \pm standard deviation. Statistical significance is determined via one-way analysis of variance (ANOVA) with a posthoc, Bonferroni's multiple comparison test (GraphPad Prism 5, La Jolla, CA). Statistical significance is marked as * $(p<0.05), * *(p<0.01)$, or ${ }^{* * * * * *}(p<0.001)$.

\section{Results \& discussion}

\section{Characterization of CDM}

A confluent cell layer of Col I-293 T-DK cell line on petri-dish was observed under phase contrast microscope (Fig. 1a, top). After decellularization, it was clear that the original cellular morphology were completely removed (Fig. 1a, bottom). The resulting extracellular matrix (ECM) layer is named cell-derived extracellular matrix $(\mathrm{CDM})$. This decellularization procedure is supposed to get rid of cellular components, specifically nucleic and cytosolic ones while retaining bioactive extracellular compositions [18, 19]. Total protein content and DNA amount before and after decellularization were examined, respectively and compared with each other. BCA assay found out that total protein amount of $\mathrm{CDM}$ is $1918 \mu \mathrm{g} /$ per dish, and there is little difference compared to that before decellularization $(1996 \mu \mathrm{g})$ (Fig. 1b). In addition, it was obvious that cellular DNA was almost completely removed by current decellularization; the amount of DNA (1.5 ng DNA/mg) in CDM was less than $2 \%$ that of the cells $(88.61 \mathrm{ng} \mathrm{DNA} / \mathrm{mg})$ before decellularization (Fig. 1c). Current DNA amount seems to be negligible, based on the report by Crapo and Gilbert; less than $50 \mathrm{ng}$ of double stranded DNA

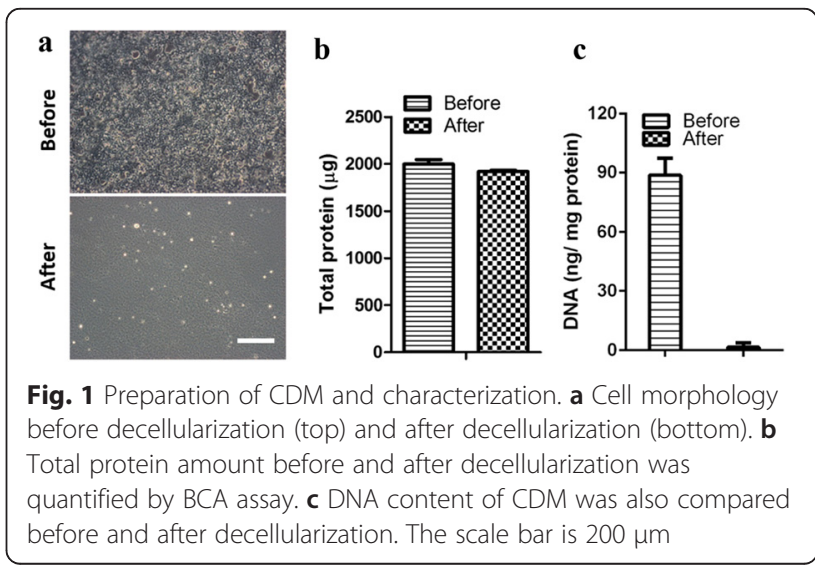


per mg of ECM should be a minimum criteria regarding the procedure of decellularization [20]. These results demonstrate that our decellularization procedure is effective in clearing cellular DNA while preserving the extracellular macromolecules to a large extent.

\section{Surface analysis of CDM-coated mesh scaffold}

To confirm whether CDM is successfully coated on the mesh scaffolds, they were examined using SEM and immunofluorescence staining against FN. The SEM images of mesh scaffold without coating exhibited smooth and randomly aligned fibers whereas both CDM-coated and FN-coated group showed the presence of coating material on the microfibers (Fig. 2); FN-coated one shows a thin layer of matrix coating on the microfibers whereas CDM-coated one holds more abundant matrix moieties in the mesh fibers. More specifically, the ECM on the CDM-coated mesh adheres to the microfibers and it also occupies the interstices in the mesh scaffold, contrasted o the ECM on the FN-mesh scaffold. The presence of ECM on mesh scaffold is further confirmed by FN immunostaining, as FN is one of the most important components of CDM [21]. Mesh scaffold alone shows no positive signals of FN. Compared to the FN-coated microfiber, CDM-coated one exhibited much better FN positive signals that homogeneously distributed through mesh scaffold (Fig. 2, Inset). Current data demonstrate that $\mathrm{CDM}$ coating is effective and rather homogeneous through the mesh scaffold.

\section{UCB-MSCs viability and proliferation}

To evaluate the capacity of the CDM-coated mesh scaffolds for cell attachment and growth, we cultured UCBMSCs and examined cell viability via Live \& Dead staining after 2 days post-seeding. Representative images of UCB-MSCs exhibited that they are well attached to both CDM- and FN-coated mesh scaffold and the cells are viable (stained in green) in these scaffolds (Fig. 3a). As the proliferation of UCB-MSCs is further examined for up to 5 days via CCK-8 assay, the cell number continuously

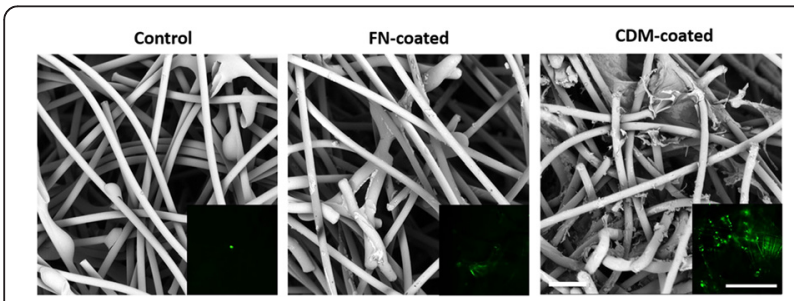

Fig. 2 Surface characterization of mesh scaffolds. The surface morphology of differently treated mesh scaffolds (control, FN-coated, and CDM-coated) is observed via SEM. The scale bar is $500 \mu \mathrm{m}$. Insets exhibit the distribution of fibronectin (green) in the mesh scaffolds. The scale bar is $200 \mu \mathrm{m}$

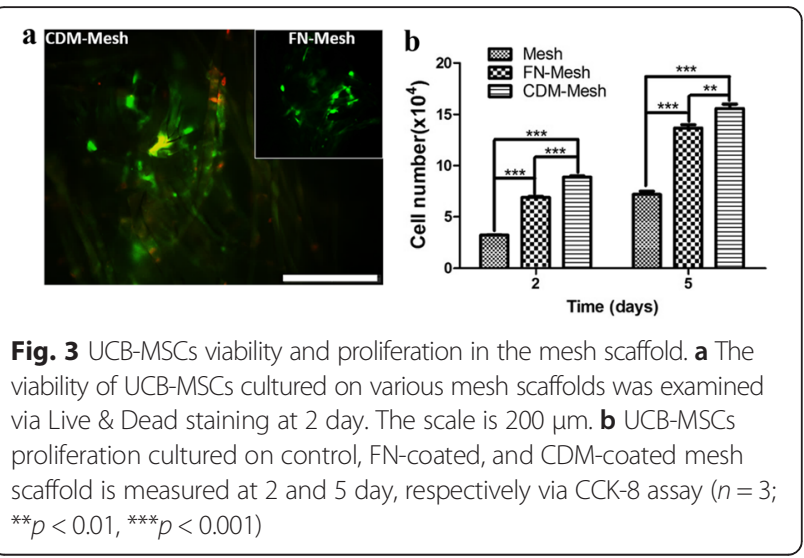

increased with time on all the mesh scaffolds. However, CDM-mesh was a significantly better on UCB-MSCs proliferation than FN-mesh and plain mesh (Fig. 3b). It can be explained that, comprised of various ECM macromolecules [22, 23], CDM not only provides abundant cell-binding motifs but also plays a certain role in promoting cell proliferation [22].

\section{ALP activity and gene expression of osteogenic markers}

The effect of CDM-coated mesh scaffolds in facilitating MSCs differentiation is examined by culturing UCBMSCs in osteogenic medium for 1 and 3 weeks, respectively. When the samples were subjected to ALP staining at 3 weeks, CDM-coated mesh was much more positive in ALP activity (Fig. 4a). Although ALP activity increased with time for all the groups, a significantly higher level of ALP activity was observed at both 1 and 3 weeks from the cells cultured on the CDM-coated mesh than those cultured on FN-mesh or control (Fig. 4b). To further examine UCB-MSCs osteogenic differentiation in gene level, Col I, ALP, OC, and BSP are used as osteogenic markers (Fig. 5). Expression levels of both ALP and BSP are relatively higher at 1 week in FNmesh and CDM-mesh group compared to that of plain mesh. However, the expression level of BSP and ALP was significantly up-regulated at 3 weeks only in CDMmesh, more than 3-fold greater than that of the other two groups. Similarly, the expression of Col I and OC was also up-regulated in the CDM-coated mesh with time. These results indicate that $\mathrm{CDM}$ can promote UCB-MSCs osteogenic differentiation in 3D environment and are better than FN.

\section{Histological analysis}

Osteogenic differentiation of UCB-MSCs on various mesh scaffolds was also analyzed by Alizarin red and von kossa staining, respectively. While Alizarin red staining exhibited a sign of calcium deposition (red color) in the CDM- 

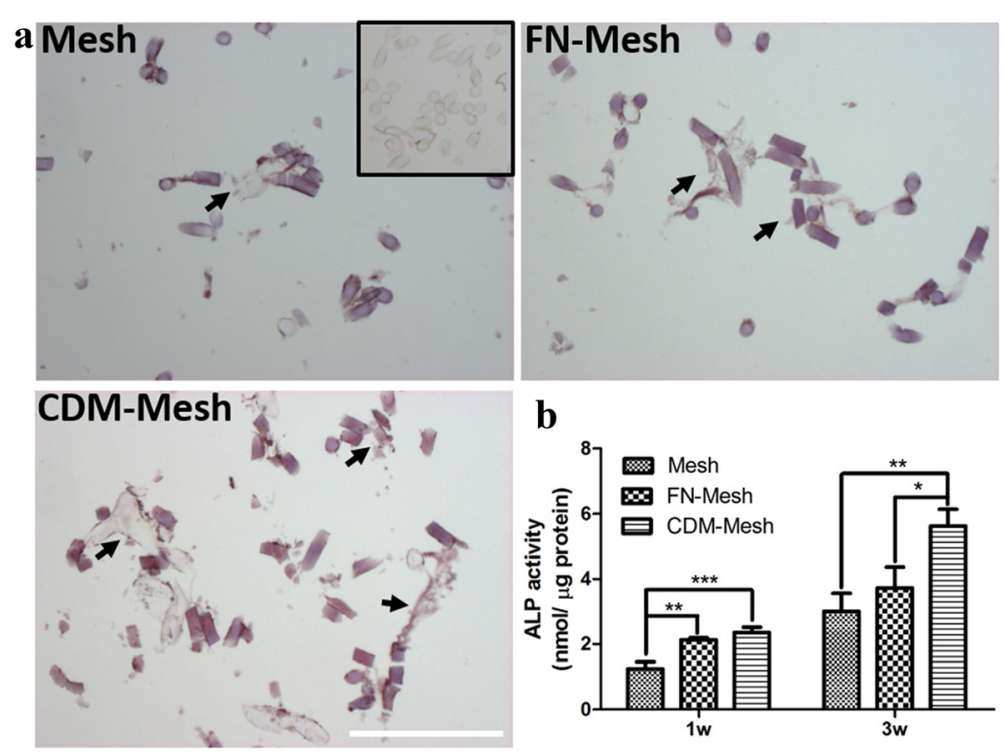

b

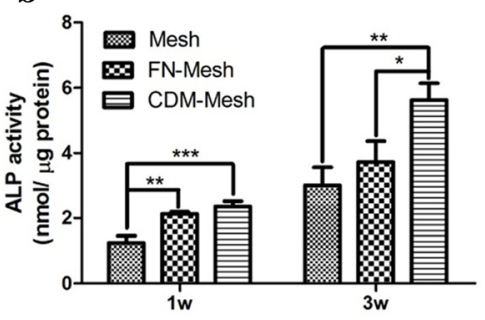

Fig. 4 Alkaline phosphatase (ALP) staining and ALP activity measurement. a UCB-MSCs cultured in osteogenic medium for 3 weeks were subjected to ALP staining. Insets show the background staining. The scale is $200 \mu \mathrm{m}$. b When the ALP activity of UCB-MSCs is also measured, that of CDM-coated mesh scaffold is significantly higher than the other groups at 3 week $\left({ }^{*} p<0.05,{ }^{* *} p<0.01,{ }^{* * *} p<0.001\right)$

mesh, both FN-mesh and control presented few positive signals (Fig. 6a). Von kossa staining also confirms much better calcium accumulation (black color) with the CDMmesh, and some positive signals were found on the FNmesh at 3 week (Fig. 6b). Insets exhibit the corresponding staining of each group at 1 week.

\section{Conclusions}

In this study, CDM obtained from in vitro cultured Col I-overexpression cells was collected and successfully coated onto 3D mesh scaffold. CDM provides a much better microenvironment for UCB-MSCs adhesion and proliferation than FN. More importantly, CDM-coated

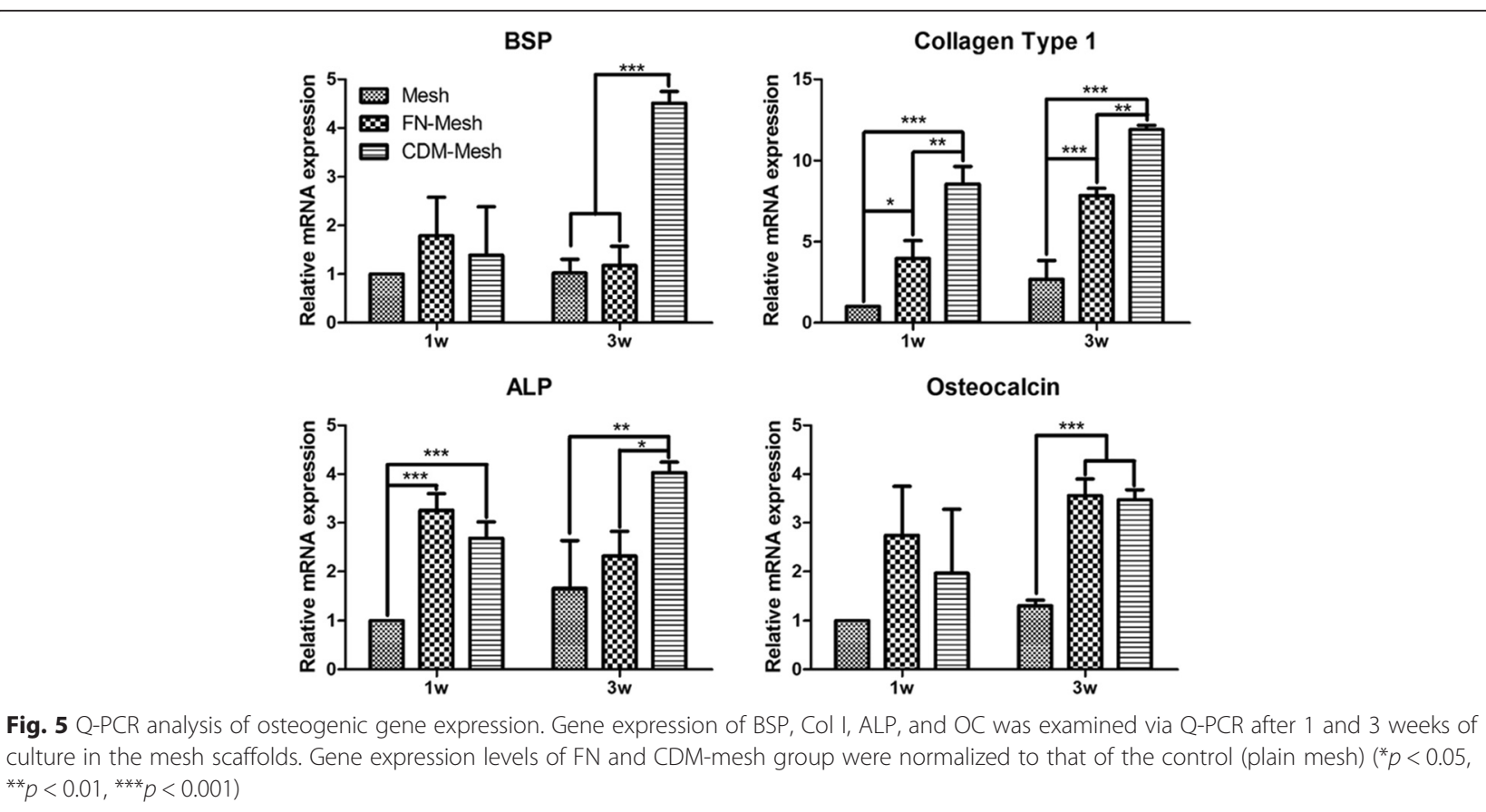



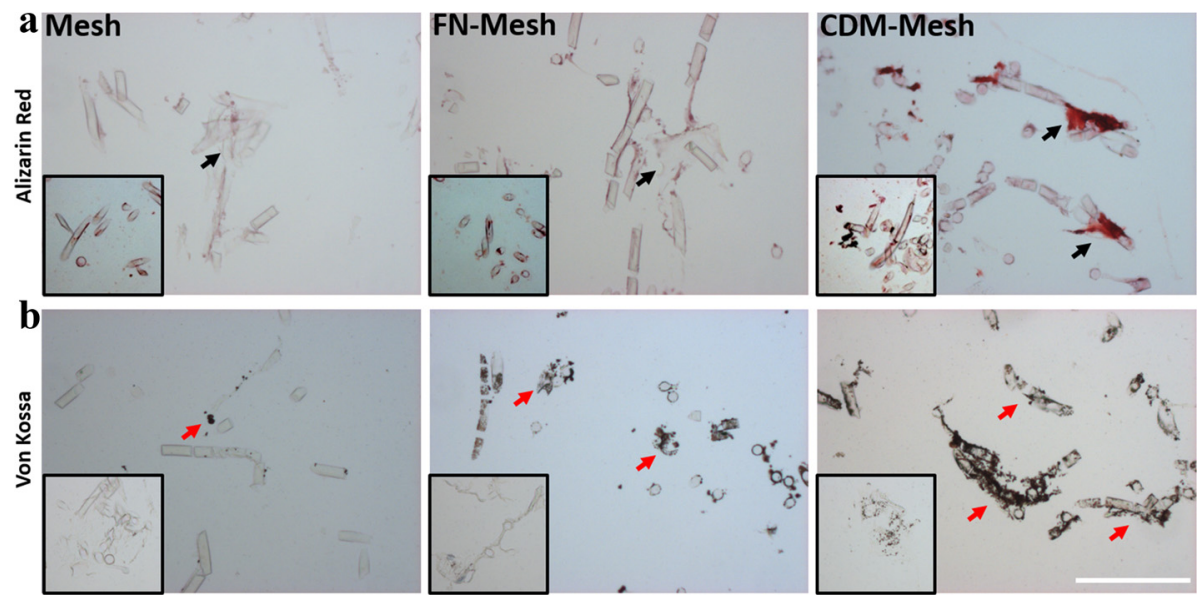

Fig. 6 Histological analysis. a UCB-MSCs cultured on the mesh scaffolds were visualized by Alizarin red staining and $\mathbf{b}$ von kossa staining at 3 week, respectively. Mineralized matrix deposition was observed for both stainings, particularly more intense in the CDM-mesh scaffold. Insets are the samples stained at 1 week. The scale is $200 \mu \mathrm{m}$

mesh scaffold supports UCB-MSCs osteogenic differentiation, much better than FN-coated one as indicated by protein and gene expression as well as by histological staining. However, the related cellular and molecular mechanism behind how CDM up-regulates the osteogenic differentiation of UCB-MSCs warrants further investigation. In summary, combination of CDM and polymer mesh scaffold can produce a biomimetic 3D microenvironment and make it a suitable platform for further investigation of stem cell culture and differentiation.

\section{Competing interests}

The authors declare that they have no competing interests.

\section{Authors' contributions}

KP and IGK developed the concept and designed experiments. YKN performed the whole process of experiments. JK provided the scaffolds and SWK provided the UCB-MSCS. PD and KP extensively contributed on the manuscript preparation. All authors read and approved the final manuscript.

\section{Acknowledgements}

This work was supported by an intramural grant 2E25260(KIST) from the Ministry of Science, ICT and Future Planning, Republic of Korea, and by a grant of the Korean Health Technology R\&D Project, Ministry of Health \& Welfare, Republic of Korea (A120216)

\section{Author details}

${ }^{1}$ Center for Biomaterials, Korea Institute of Science and Technology, Seoul 02792, Republic of Korea. ${ }^{2}$ Department of Biomedical Science, Kyung Hee University, Seoul 02447, Republic of Korea. ${ }^{3}$ Department of Technical application, Korea Institute of Industrial Technology, Gyeonggi 31056, Republic of Korea. ${ }^{4}$ Department of Biochemistry and Molecular Biology, Asan Medical Center, University of Ulsan College of Medicine, Seoul 05505, Republic of Korea. ${ }^{5}$ Department of Biomedical engineering, University of Science and Technology, Daejeon 34113, Republic of Korea.

Received: 31 December 2015 Accepted: 7 March 2016

Published online: 07 April 2016

\section{References}

1. Langer R, Vacanti JP. Tissue engineering. Science. 1993;260:920-6.
2. Karageorgiou V, Kaplan D. Porosity of $3 D$ biomaterial scaffolds and osteogenesis. Biomaterials. 2005;26:5474-91.

3. Jiang Y, Chen L, Zhang S, Tong T, Zhang W, Liu W, et al. Incorporation of bioactive polyvinylpyrrolidone-iodine within bilayered collagen scaffolds enhances the differentiation and subchondral osteogenesis of mesenchymal stem cells. Acta Biomater. 2013;9:8089-98.

4. Whu SW, Hung K-C, Hsieh K-H, Chen C-H, Tsai C-L, S-h H. In vitro and in vivo evaluation of chitosan-gelatin scaffolds for cartilage tissue engineering. Mater Sci Eng C. 2013:33:2855-63.

5. Zhou H, Xu HHK. The fast release of stem cells from alginate-fibrin microbeads in injectable scaffolds for bone tissue engineering. Biomaterials. 2011;32:7503-13.

6. Wu C, Zhang Y, Zhou Y, Fan W, Xiao Y. A comparative study of mesoporous glass/silk and non-mesoporous glass/silk scaffolds: Physiochemistry and in vivo osteogenesis. Acta Biomater. 2011;7:2229-36.

7. Jang $\mathrm{CH}$, Cho YB, Choi CH, Jang YS, Jung W-K, Lee H, et al. Effect of umbilical cord serum coated 3D PCL/alginate scaffold for mastoid obliteration. Int J Pediatr Otorhinolaryngol. 2014;78:1061-5.

8. Hynes RO. Extracellular matrix: not just pretty fibrils. Science. 2009;326:1216-9.

9. Lutolf MP. Integration column: artificial ECM: expanding the cell biology toolbox in 3D. Integr Biol. 2009;1:235-41.

10. Frantz C, Stewart KM, Weaver VM. The extracellular matrix at a glance. J Cell Sci. 2010;123:4195-200.

11. Lu H, Hoshiba T, Kawazoe N, Koda I, Song M, Chen G. Cultured cell-derived extracellular matrix scaffolds for tissue engineering. Biomaterials. 2011;32: 9658-66.

12. Guilak F, Cohen DM, Estes BT, Gimble JM, Liedtke W, Chen CS. Control of stem cell fate by physical interactions with the extracellular matrix. Cell Stem Cell. 2009:5:17-26.

13. Kang Y, Kim S, Bishop J, Khademhosseini A, Yang Y. The osteogenic differentiation of human bone marrow MSCS on HUVEC-derived ECM and $\beta$-TCP scaffold. Biomaterials. 2012;33:6998-7007.

14. Bae SE, Bhang SH, Kim B-S, Park K. Self-assembled extracellular macromolecular matrices and their different osteogenic potential with preosteoblasts and rat bone marrow mesenchymal stromal cells. Biomacromolecules. 2012;13:2811-20.

15. Choi D, Suhaeri M, Hwang M, Kim I, Han D, Park K. Multi-lineage differentiation of human mesenchymal stromal cells on the biophysical microenvironment of cell-derived matrix. Cell Tissue Res. 2014;357:781-92.

16. Lu F-Z, Fujino M, Kitazawa Y, Uyama T, Hara Y, Funeshima N, et al. Characterization and gene transfer in mesenchymal stem cells derived from human umbilical-cord blood. J Lab Clin Med. 2005;146:271-8.

17. Choi YS, Im MW, Kim CS, Lee MH, Noh SE, Lim SM, et al. Chondrogenic differentiation of human umbilical cord blood-derived multilineage progenitor cells in atelocollagen. Cytotherapy. 2008;10:165-73. 
18. Hoganson DM, Owens GE, O'Doherty EM, Bowley CM, Goldman SM, Harilal $\mathrm{DO}$, et al. Preserved extracellular matrix components and retained biological activity in decellularized porcine mesothelium. Biomaterials. 2010;31:6934-40.

19. Fitzpatrick JC, Clark PM, Capaldi FM. Effect of decellularization protocol on the mechanical behavior of porcine descending aorta. International Journal of Biomaterials. 2010;62053:11.

20. Crapo PM, Gilbert TW, Badylak SF. An overview of tissue and whole organ decellularization processes. Biomaterials. 2011;32:3233-43.

21. Cha MH, Do SH, Park GR, Du P, Han K-C, Han DK, et al. Induction of redifferentiation of passaged rat chondrocytes using a naturally obtained extracellular matrix microenvironment. Tissue Eng A. 2013;19:978-88.

22. Chen X-D. Extracellular matrix provides an optimal niche for the maintenance and propagation of mesenchymal stem cells. Birth Defects Research Part C: Embryo Today: Reviews. 2010;90:45-54.

23. Reilly GC, Engler AJ. Intrinsic extracellular matrix properties regulate stem cell differentiation. J Biomech. 2010;43:55-62.

Submit your next manuscript to BioMed Central and we will help you at every step:

- We accept pre-submission inquiries

- Our selector tool helps you to find the most relevant journal

- We provide round the clock customer support

- Convenient online submission

- Thorough peer review

- Inclusion in PubMed and all major indexing services

- Maximum visibility for your research

Submit your manuscript at www.biomedcentral.com/submit
Biomed Central 